\title{
LOCALIZATION AND SUMMABILITY OF MULTIPLE HERMITE SERIES
}

\section{G. E. KARADZHOV and E. E. EL-ADAD}

Institute of Mathematics

Bulgarian Academy of Sciences

1113 Sofia, BULGARIA

(Received April 10, 1995 and in revised form October 5, 1995)

\begin{abstract}
The multiple Hermite series in $\mathbf{R}^{n}$ are investigated by the Riesz summability method of order $\alpha>(n-1) / 2$. More precisely, localization theorems for some classes of functions are proved and sharp sufficient conditions are given. Thus the classical Szegö results are extended to the $n$-dimensional case. In particular, for these classes of functions the localization principle and summability on the Lebesgue set are established.
\end{abstract}

KEY WORDS AND PHRASES: Riesz summability, multiple Hermite series 1991 AMS SUBJECT CLASSIFICATION CODES: 42C10

\section{Statement of the main results}

Let $f$ be locally in $L^{1}\left(\mathbf{R}^{n}\right), n \geq 2$, and consider the multiple Hermite series

$$
f(y) \sim \sum f_{k} e^{-y^{2} / 2} \tilde{H}_{k}(y), f_{k}=\int_{\mathbf{R}^{n}} f(y) e^{-y^{2} / 2} \tilde{H}_{k}(y) d y,
$$

where $\tilde{H}_{k}(y)=\tilde{H}_{k_{1}}\left(y_{1}\right) \ldots \tilde{H}_{k_{n}}\left(y_{n}\right), k=\left(k_{1}, . ., k_{n}\right), k_{\imath} \geq 0, y=\left(y_{1}, . ., y_{n}\right)$, is a product of the normalized Hermitian polynomials. Here and later on $y^{2}$ stands for the scalar product $(y, y)$ in $\mathbf{R}^{n}$ and for simplicity we shall write $x y$ instead of $(x, y)$. The corresponding spherical partial sum has the form

$$
E_{\lambda} f(y)=\sum_{\mu_{k}<\lambda} f_{k} \phi_{k}(y)
$$

where $\mu_{k}=2|k|+n$ and $\phi_{k}(x)=e^{-x^{2} / 2} \tilde{H}_{k}(x)$ are the eigenvalues and orthonormalized eigenfunctions of the operator $A=-\Delta+x^{2}$ in $L^{2}\left(\mathbf{R}^{n}\right)$. Let

$$
E_{\lambda}^{\alpha} f(y)=\sum_{\mu_{k}<\lambda}\left(1-\mu_{k} / \lambda\right)^{\alpha} f_{k} \phi_{k}(y)
$$

be the corresponding Riesz means of order $\alpha>0$. We shall prove the convergence

$$
R_{\lambda}^{\alpha} \stackrel{\text { def }}{=} E_{\lambda}^{\alpha} f(y)-\int_{|x-y|<\delta} f(x) I^{\alpha} e(\lambda, x, y) d x=o(1)
$$

as $\lambda \rightarrow+\infty$, locally uniformly with respect to $y$, where $\delta>0$ and

$$
I^{\alpha} e(\lambda, x, y)=\int_{0}^{\lambda}(1-\mu / \lambda)^{\alpha} d e(\mu, x, y)
$$

is the Riesz kernel, under some conditions at infinity for the function $f$, including a system of sharp sufficient conditions. Thus the classical Szegö results [18] are extended to the n-dimensional 
case. In particular, for these classes of functions the localization principle and summability on the Lebesgue set are established. For other results see, for example, [1]-[4], [6]-[8], [10]-[19] and the bibliography in [15], [19]. Here

$$
e(\lambda, x, y)=\sum_{\mu_{k}<\lambda} \phi_{k}(x) \phi_{k}(y)
$$

is the spectral function of $A$.

In stating the main results we use the following notations. Let $a(\lambda, x)$ be the characteristic function of the set $\left\{x \in \mathbf{R}^{n}: A<x^{2}<\lambda-\lambda^{1 / 3}\right\}$ and $b(\lambda, x)$ - the characteristic function of the set $\left\{x \in \mathbf{R}^{n}:\left|x^{2}-\lambda\right|<\lambda^{1 / 3+\epsilon}\right\}$ for some small $\epsilon>0$ and large $A>0$.

Theorem 1. If $\alpha>(n-1) / 2$ and

$$
\begin{gathered}
\int_{\mathbf{R}^{n}} a(\lambda, x)\left(1-x^{2} / \lambda\right)^{-1 / 4}|x|^{-(n+1) / 2-\alpha}|f(x)| d x=o\left(\lambda^{\alpha / 2-(n-1) / 4}\right) \\
\int_{\mathbf{R}^{n}} b(\lambda, x)|f(x)| d x=o\left(\lambda^{\alpha+1 / 3}\right)
\end{gathered}
$$

then the convergence relation (1.1) is fulfilled.

Remark 1. The condition $\left(H_{2}\right)$ is exact. Namely, it is satisfied by the function $f(x)=$ $|x|^{\beta}, \beta>0$ for every $\beta<2 \alpha-n+2$, but not for $\beta \geq 2 \alpha-n+2$. On the other hand, $R_{\lambda}^{\alpha} f(0)$ is divergent if $\beta \geq 2 \alpha-n+2, \alpha>(n-1) / 2$.

For the functions which are differentiable at infinity we can improve the condition $\left(H_{1}\right)$.

Theorem 2 Let the function $f$ be differentiable at infinity and satisfy for $\alpha>(n-1) / 2$ the condition $f(x)=O\left(|x|^{\beta}\right)$ as $|x| \rightarrow \infty$ for $\beta<2 \alpha-n+2$ and

$$
\int_{\mathbf{R}^{n}} a(\lambda, x)\left(1-x^{2} / \lambda\right)^{-3 / 4}|x|^{-(n+1) / 2-\alpha-1}|\nabla f(x)| d x=o\left(\lambda^{\alpha / 2-(n-1) / 4}\right) .
$$

Then the convergence relation (1.1) is valid.

Corollary 1. Let the function $f$ be differentiable at infinity and $f, \nabla f=O\left(|x|^{\beta}\right)$ as $|x| \rightarrow \infty$, where $\beta<2 \alpha-n+2, \alpha>(n-1) / 2$. Then the relation (1.1) is true.

It is natural to "interpolate" between conditions $\left(H_{1}\right)$ and $\left(H_{1}^{\prime}\right)$. Define

$$
\omega(x, f)=\sum_{\imath=1}^{n} \sup _{0 \leq h_{\imath} \leq 1}\left|f(x+H)-f\left(x+H_{\imath}\right)\right|
$$

where $H=\left(h_{1}, . ., h_{n}\right), H_{\imath}=\left(h_{1}, . ., h_{2-1}, 0, h_{\imath+1}, . ., h_{n}\right)$.

Theorem 3. Let the function $f$ satisfy for $\alpha>(n-1) / 2$ the condition $f(x)=O\left(|x|^{\beta}\right)$ as $|x| \rightarrow \infty$ for $\beta<2 \alpha-n+2$ and

$$
\int_{\mathbf{R}^{n}} a(\lambda, x)\left(1-x^{2} / \lambda\right)^{-3 / 4}|x|^{-(n+1) / 2-\alpha} \omega(x, f) d x=o\left(\lambda^{\alpha / 2-(n-1) / 4}\right) .
$$

Then the convergence relation (1.1) is fulfilled.

Remark 2. The conditions of theorem 3 are satisfied by the function $f(x)=|x|^{\beta}, \beta>0$, if $\beta<2 \alpha-n+2$, and they are not satisfied if $\beta \geq 2 \alpha-n+2$. Therefore, according to remark 1 , theorem 3 provides a system of sharp sufficient conditions.

Corollary 2 (localization principle). Let $y \in R^{n}, \delta>0$ be fixed. Then under the conditions of theorems $1,2,3$ respectively we have

$$
E_{\lambda}^{\alpha} f(y) \rightarrow 0 \text { if } f(x)=0 \text { for }|x-y|<\delta .
$$

As a consequence of theorems $1,2,3,4$ and corollary 4.16 [16] we obtain

Corollary 3. Under the conditions of theorems $1,2,3$ respectively we have $E_{\lambda}^{\alpha} f(y) \rightarrow f(y)$ on the Lebesgue set of the function $f$.

The further organisation of the paper is as follows. The results about the asymptotics of the Riesz kernels are formulated in section 2, while the proofs are given in sections 7-10. These asymptotics are used to prove theorems 1-3 in sections 3-5 respectively. Finally, remark 1 is proved in section 6 . 


\section{Asymptotics of Riesz kernels}

Here we state the uniform asymptotics of the Riesz kernels which we need. Since

$$
E_{\lambda}^{\alpha} f(y)=\int_{\mathbf{R}^{n}} I^{\alpha} e(\lambda, x, y) f(x) d x, \alpha>0,
$$

we have to find the asymptotics or bounds for the Riesz kernels $I^{\alpha} e(\lambda, x, y)$ as $\lambda \rightarrow \infty$, which must be uniform with respect to the parameters $x \in \boldsymbol{R}^{n}, y^{2}<A$. It is convenient to consider also the functions

$$
e_{\alpha}(\lambda, x, y)=\lambda^{\alpha} I^{\alpha} e(\lambda, x, y), E_{\alpha}(\lambda, x, y)=e_{\alpha}(\lambda, \sqrt{\lambda} x, \sqrt{\lambda} y) .
$$

Theorem 4. If $x^{2}+y^{2}<A$ and $\alpha \geq(n-1) / 2$ then

$$
\left|I^{\alpha} e(\lambda, x, y)-I^{\alpha} e^{o}(\lambda, x, y)\right| \leq c \lambda^{(n-1) / 2} G_{\alpha}(\sqrt{\lambda}|x-y|),
$$

where

$$
G_{\alpha}(s)=(1+s)^{-(n+1) / 2-\alpha}, s \geq 0, e^{o}(\lambda, x, y)=(2 \pi)^{-n} \int_{\xi^{2} \leq \lambda} e^{\imath(x-y) \xi} d \xi
$$

and for $d_{\alpha}=(2 \pi)^{-n / 2} 2^{\alpha} \Gamma(\alpha+1)$,

$$
I^{\alpha} e^{0}(\lambda, x, y)=\lambda^{n / 2} F_{\alpha}(\sqrt{\lambda}|x-y|), F_{\alpha}(s)=d_{\alpha} s^{-n / 2-\alpha} J_{n / 2+\alpha}(s) .
$$

Theorem 5. Let $A / \lambda<x^{2}<1-\delta,|y|<\epsilon|x|$ and $\alpha>0$. Then for every small $\delta>0, \epsilon>0$ and $A>0$ we have the uniform asymptotics

$$
E_{\alpha}(\lambda, x, y)=\lambda^{-1 / 2} \sum_{k=1}^{4} b_{k}(\lambda, x, y, \alpha) e^{2 \lambda \psi_{k}}+|x|^{-(n+1) / 2-\alpha} O\left(\lambda^{-1}\right)
$$

where

$$
\left|b_{k}\right| \leq c|x|^{-(n+1) / 2-\alpha},\left|\nabla_{x} b_{k}\right| \leq c|x|^{-(n+1) / 2-\alpha-1}
$$

and

$$
\left|\nabla \psi_{k}\right|^{2}=1-x^{2},\left|\Delta \psi_{k}\right|^{2} \leq c\left(1-x^{2}\right)^{-1} .
$$

Theorem 6. There exist $\delta>0, \epsilon>0$ such that the uniform asymptotics

$$
E_{\alpha}(\lambda, x, y)=\sum_{k=0}^{\infty}\left(a_{1 k}(\lambda, x, y) \lambda^{-k-1 / 3}+b_{1 k}(\lambda, x, y) \lambda^{-k-2 / 3}\right)
$$

holds if $\left|x^{2}-1\right|<\delta,|y|<\epsilon|x|$, where

$$
a_{1 k}=\left(a_{k} e^{\lambda A}+b_{k} e^{-\lambda A}\right) A i\left(\lambda^{2 / 3} B\right), b_{1 k}=\left(c_{k} e^{\lambda A}+d_{k} e^{-\lambda A}\right) A i^{\prime}\left(\lambda^{2 / 3} B\right)
$$

and the functions $\lambda \rightarrow a_{k}, b_{k}, c_{k}, d_{k}$, or their derivatives with respect to $x$ are bounded. Here $A i$ is the Airy function and the smooth functions $A=A(x, y), B=B(x, y)$ have the following properties: $\operatorname{Re} A=0, \operatorname{Im} B=0$. Moreover, let $x=|x| \omega$ and

$$
a^{2}=\left(1-y^{2}\right)\left(1-(\omega y)^{2}\right)^{-1}
$$

Then

$$
\begin{gathered}
B(x, y)<0 \text { if } x^{2}<a^{2}, \\
B(x, y) \sim c(y, \omega)\left(x^{2}-a^{2}\right) \text { as } x^{2} \rightarrow a^{2}, c(y, \omega)>0 .
\end{gathered}
$$

From theorem 6 , the asymptotics of the Airy function and $(2.8),(2.9)$ it follows 
Corollary 4. There exists $\delta>0$ such that

$$
E_{\alpha}(\lambda, x, y)=\lambda^{-1 / 2} \sum_{k=1}^{4}\left(a_{k}\left(a^{2}-x^{2}\right)^{-1 / 4}+b_{k}\left(a^{2}-x^{2}\right)^{1 / 4}\right) \exp i \lambda \psi_{k}+\left(a^{2}-x^{2}\right)^{-1} O\left(\lambda^{-1}\right)
$$

uniformly with respect to $x, y$ if $1-\delta<x^{2}<1-\lambda^{-2 / 3+\epsilon}, y^{2}<A / 2 \lambda$, where $\epsilon>0, A>0$ are fixed The functions $\lambda \rightarrow a_{k}, b_{k}$ and their derivatives over $x$ are bounded and $\psi_{k}$ satisfy (2.5).

Theorem 7. Let $x^{2}>1+\delta, y^{2}<\epsilon$. Then we have the uniform estimate

$$
\left|E_{\alpha}(\lambda, x, y)\right| \leq c\left(x^{2}-1\right)^{-1 / 4} \lambda^{-1 / 2} \exp \left(-c \delta \lambda\left(x^{2}-1\right)^{1 / 2}\right),
$$

where $c$ is a positive constant.

As a consequence of theorems 6 and 7 it follows

Corollary 5. If $x^{2}>\lambda+\lambda^{1 / 3+\epsilon}, \epsilon>0, y^{2}<A$, then

$$
\left|I^{\alpha} e(\lambda, x, y)\right| \leq c \lambda^{-\alpha-1 / 3} \exp \left(-c \lambda^{1 / 3}\left(x^{2} / \lambda-1\right)^{1 / 2}\right) .
$$

\section{Proof of theorem 1}

Let $y^{2}<A / 2, \delta>0, \alpha>(n-1) / 2, n \geq 2$, According to (1.1) and (2.1)

$$
R_{\lambda}^{\alpha} f(y)=\int_{|x-y|>\delta} f(x) I^{\alpha} e(\lambda, x, y) d x .
$$

From theorem 4 and the asymptotics of the Bessel functions it follows

$$
R_{\lambda}^{\alpha} f(y)=\int_{x^{2}>A} f(x) I^{\alpha} e(\lambda, x, y) d x+o(1) .
$$

Therefore it is sufficient to prove the relations

$$
K_{j}(\lambda, y)=\int_{\mathbf{R}^{n}} a_{\jmath}(\lambda, x) f(x) I^{\alpha} e(\lambda, x, y) d x=o(1),
$$

for $1 \leq j \leq 4, y^{2}<A / 2, \alpha>(n-1) / 2, n \geq 2$, where $a_{1}(\lambda, x)$ is the characteristic function of the set $\left\{x \in \mathbf{R}^{n}: A<x^{2}<\lambda(1-\delta)\right\}, a_{2}(\lambda, x)$ - the characteristic function of the set $\left\{x \in \mathbf{R}^{n}:(1-\delta) \lambda<x^{2}<\lambda-\lambda^{1 / 3+\epsilon}\right\}, a_{3}(\lambda, x)=b(\lambda, x)$ and $a_{4}(\lambda, x)$ is the characteristic function of the set $\left\{x \in \mathbf{R}^{n}: x^{2}>\lambda+\lambda^{1 / 3+\epsilon}\right\}$ for some small $\epsilon>0, \delta>0$.

a. Estimate of $K_{1}$. It is not hard to see that theorem 5 implies the bound

$$
\left|I^{\alpha} e(\lambda, x, y)\right| \leq c\left(1-x^{2} / \lambda\right)^{-1 / 4}|x|^{-(n+1) / 2-\alpha} \lambda^{(n-1) / 4-\alpha / 2}
$$

if $A<x^{2}<(1-\delta) \lambda, y^{2}<A / 2, \alpha>0$. So the hypothesis $\left(H_{1}\right)$ gives (3.2) for $K_{1}$.

b. Estimate of $K_{2}$. Now we can use corollary 4 . Since $a^{2}-x^{2} / \lambda>\left(1-x^{2} / \lambda\right) / 2$ for large $\lambda$ we see that the estimate (3.3) is fulfilled if $(1-\delta) \lambda<x^{2}<\lambda-\lambda^{1 / 3+\epsilon}, y^{2}<A / 2$. Thus $\left(H_{1}\right)$ shows (3.2) for $K_{2}$.

c. Estimate of $K_{3}$. From theorem 6 and $\left(H_{2}\right)$ we get (3.2) for $K_{3}$.

d. Estimate of $K_{4}$. Using corollary 5 we obtain

$$
\begin{gathered}
\left|I^{\alpha} e(\lambda, x, y)\right| \leq c \lambda^{-\alpha-1 / 3} \exp \left(-c \lambda^{\epsilon / 2}\right) \text { if } x^{2}>\lambda+\lambda^{1 / 3+\epsilon} \\
\left|I^{\alpha} e(\lambda, x, y)\right| \leq c \lambda^{-\alpha-1 / 3} \exp \left(-c|x|^{1 / 2}\right) \text { if } x^{2}>\lambda^{2} .
\end{gathered}
$$

On the other hand $\left(H_{1}\right)$ gives $\int_{|x|>1}|x|^{-N}|f(x)| d x<\infty$ for large $N$, so the last three estimates and $\left(H_{1}\right)$ imply (3.2) for $K_{4}$. Theorem 1 is proved. 


\section{Proof of theorem 2}

As in the proof of theorem 1 we have to estimate the integrals $K_{\jmath}(\lambda, y)$ given by (3.2) for $y^{2}<A / 2$. It is clear that the estimate (3.2) for $K_{3}$ and $K_{4}$ are valid again. Thus it remains to bound $K_{1}$ and $K_{2}$. Consider also the integrals $(j=1,2)$ :

$$
B_{\jmath}(\lambda, y)=\lambda^{n / 2-\alpha} \int_{\mathbf{R}^{n}} a_{\jmath}(\lambda, \sqrt{\lambda} x) f(\sqrt{\lambda} x) E_{\alpha}(\lambda, x, y) d x .
$$

a. Estimate of $K_{1}$. According to theorem 5 we have the following asymptotics for $\alpha>0$

$$
E_{\alpha}(\lambda, x, y)=\lambda^{-1 / 2} \sum_{k=1}^{4} b_{k} e^{2 \lambda \psi_{k}}+|x|^{-(n+1) / 2-\alpha} O\left(\lambda^{-1}\right)
$$

uniformly in the domain $\left\{x, y \in \mathbf{R}^{n}: A / \lambda<x^{2}<1-\delta, y^{2}<A / 2 \lambda\right\}$, where $b_{k}$ satisfy (2.4).

Using the estimate $f(x)=O\left(|x|^{\beta}\right), \beta>0$ as $|x| \rightarrow \infty$, we obtain for $\alpha>(n-1) / 2$ :

$$
\begin{gathered}
B_{1}(\lambda, y)=\lambda^{(n-1) / 2-\alpha} \sum_{k=1}^{4} \int_{\mathbf{R}^{n}} e^{2 \lambda \psi_{k}} a_{1} b_{k} f(\sqrt{\lambda} x) d x+ \\
O\left(\lambda^{\beta / 2+n / 2-\alpha-1} \log \lambda+\lambda^{-1 / 2}\right) .
\end{gathered}
$$

Let $I(\lambda)$ be the integral in (4.1) together with the factor $\lambda^{(n-1) / 2-\alpha}$. We shall integrate by parts using the operator $L_{k}$, where its transpose is given by $\sum \partial_{j} \psi_{k}\left|\nabla \psi_{k}\right|^{-2} \partial_{j}, 1 \leq j \leq n$, and $\partial_{j}=\partial / \partial x_{j}$. Taking into account (2.5) we get

$$
\begin{gathered}
I(\lambda)=\lambda^{(n-1) / 4-\alpha / 2}\left(\int_{\mathbf{R}^{n}} a_{1}(\lambda, x)|x|^{-(n+1) / 2-\alpha-1}|\nabla f(x)| d x+\lambda^{-1 / 2} B\right)+ \\
O\left(\lambda^{-1 / 2}+\lambda^{\beta / 2+n / 2-\alpha-3 / 2}\right),
\end{gathered}
$$

where

$$
\begin{gathered}
B=\int_{\mathbf{R}^{n}} a_{1}(\lambda, x)|x|^{-(n+1) / 2-\alpha-1}|f(x)| d x= \\
O\left(\lambda^{\beta / 2+(n-1) / 4-\alpha / 2-1 / 2} \log \lambda\right) .
\end{gathered}
$$

Since $\beta<2 \alpha+2-n,(4.1)-(4.3)$ and $\left(H_{1}^{\prime}\right)$ give $K_{1}=o(1)$.

b. Estimate of $K_{2}$. Using corollary 4 and $1-2 A / \lambda<a^{2}<1$ for $y^{2}<A / 2 \lambda$ and large $\lambda$ we obtain:

$$
\begin{gathered}
B_{2}(\lambda, y)=\lambda^{(n-1) / 2-\alpha} \sum_{k=1}^{4} \int_{\mathbf{R}^{n}} e^{2 \lambda \psi_{k}} a_{2}(\lambda, \sqrt{\lambda} x) g_{k} f(\sqrt{\lambda} x) d x+ \\
O\left(\lambda^{\beta / 2+n / 2-\alpha-1} \log \lambda\right)
\end{gathered}
$$

where $g_{k}=a_{k}(\lambda, x, y)\left(a^{2}-x^{2}\right)^{-1 / 4}+b_{k}(\lambda, x, y)\left(a^{2}-x^{2}\right)^{1 / 4}$. Integrating by parts as at the estimate of $K_{1}$ and taking into account $(2.5),\left(H_{1}^{\prime}\right)$ we get

$$
K_{2}=I+O\left(\lambda^{\beta / 2+n / 2-\alpha-1}\right)+o(1)
$$

where

$$
I=\int_{\mathbf{R}^{n}} a_{2}(\lambda, x)\left(1-x^{2} / \lambda\right)^{-7 / 4}|f(x)| d x O\left(\lambda^{-\alpha-3 / 2}\right),
$$

Since $\left(1-x^{2} / \lambda\right)^{-3 / 4}<\lambda^{1 / 2}$ in the integral $I$ and $f(x)=O\left(|x|^{\beta}\right)$ as $|x| \rightarrow \infty$ we find

$$
I=O\left(\lambda^{\beta / 2+n / 2-\alpha-1} \log \lambda\right) .
$$

Hence (4.5), (4.6) imply $K_{2}=o(1)$ since $\beta<2 \alpha+2-n$. Theorem 2 is proved. 


\section{Proof of theorem 3}

As in the proof of theorems 1 and 2 it is sufficient to estimate the integrals $K_{3}=K_{j}(f), 1 \leq$ $\jmath \leq 4$. For $j=3,4$ we have the bound (3.2). Further let

$$
f_{1}(x)=\int_{0}^{1} \cdots \int_{0}^{1} f(x+h) d h, f_{0}(x)=f(x)-f_{1}(x) .
$$

Then $f_{\jmath}(x)=O\left(|x|^{\beta}\right)$ as $|x| \rightarrow \infty$ for $\beta<2 \alpha-n+2$ and

$$
\left|\nabla f_{1}(x)\right| \leq \omega(x, f),\left|f_{0}(x)\right| \leq \omega(x, f),
$$

therefore $f_{0}$ satisfies $\left(H_{1}\right)$ and $f_{1}$ satisfies $\left(H_{1}^{\prime}\right)$. Evidently, $K_{\jmath}(f)=K_{\jmath}\left(f_{0}\right)+K_{\jmath}\left(f_{1}\right), j=1,2$. As in the proof of theorems 1 and 2 we obtain $K_{j}(f)=o(1), j=1,2$. Thus theorem 3 is proved.

\section{Proof of remark 1}

It is not hard to see that for $\alpha>(n-1) / 2$ remark 1 will follow from (1.1), theorem 4 , corollary $4.16[16]$ and the asymptotics

$$
E_{\lambda}^{\alpha} f(0)=\lambda^{n / 2+\beta / 2-\alpha-1}\left(a(\lambda)+O\left(\lambda^{-1}\right)\right)+O\left(\lambda^{-\beta / 2}\right),
$$

where $f(x)=|x|^{\beta}, \alpha>0, \beta>0, n \geq 2$ and $a(\lambda)=a_{+}(\lambda)+a_{-}(\lambda)+a_{o}(\lambda)$,

$$
\begin{gathered}
a_{ \pm}(\lambda)=c \sum_{k \geq 1}(-1)^{k n}| \pm \pi / 4+k \pi|^{-\alpha-1} \sin (\lambda \pi(k+1 / 4)-(\alpha+n / 2) \pi / 2) \\
a_{o}(\lambda)=c(\pi / 4)^{-\alpha-1} \sin (\lambda \pi / 4-(\alpha+n / 2) \pi / 2)
\end{gathered}
$$

$c$ being a positive constant.

To prove (6.1) we shall use the formula

$$
e_{\alpha}(\lambda, x, y)=\Gamma(\alpha+1)(2 \pi i)^{-1} \int_{S} e^{\lambda p} V(p, x, y) H_{\alpha}(\lambda+n, p) d p
$$

where $S=(\delta-i \pi / 2, \delta+i \pi / 2), \delta>0, \alpha>0$ and the function $s \rightarrow H_{\alpha}(s, p)$ is 2-periodic,

$$
H_{\alpha}(s, p)=\sum_{k=-\infty}^{+\infty} e^{\imath s k \pi}(p+i k \pi)^{-\alpha-1}, p \in S, \alpha>0 .
$$

For proving (6.2) we notice that $p^{-\alpha-1} \Gamma(\alpha+1) V(p, x, y)$ is the Laplace transform of the function $\lambda \rightarrow e_{\alpha}(\lambda, x, y)$, where

$$
V(p, x, y)=\int_{0}^{\infty} e^{-\lambda p} d e(\lambda, x, y), \operatorname{Re} p>0
$$

in particular, $V(p+i k \pi, x, y)=e^{i k \pi n} V(p, x, y)$. Applying the inverse Laplace formula we get (6.2).

Since (see, for example, [18], [19])

$$
V(p, x, y)=(2 \pi \sinh 2 p)^{-n / 2} \exp \left(-\frac{x^{2}+y^{2}}{2} \operatorname{coth} 2 p+\frac{x y}{\sinh 2 p}\right),
$$

we can write

$$
E_{\lambda}^{\alpha} f(0)=\Gamma(\alpha+1)(2 \pi i)^{-1} \lambda^{-\alpha} \int_{S} e^{\lambda p} H_{\alpha}(\lambda+n, p) u(p, 0) d p
$$

where

$$
u(p, 0)=(2 \pi \sinh 2 p)^{-n / 2} \int_{\mathbf{R}^{n}}|x|^{\beta} \exp \left(-2^{-1} x^{2} \operatorname{coth} 2 p\right) d x, \operatorname{Re} p>0 .
$$

The integrand in (6.4) has singularities only at the points $p=0, \pm i \pi / 2$ and $p= \pm i \pi / 4$. To find the asymptotics of the function (6.4) we shall apply the method of the stationary phase. Let 
$1=g_{1}(p)+g_{2}(p)+g_{3}(p)$ for $p \in S$, where $g_{\jmath} \in C^{\infty}$ and supp $g_{1} \subset\{p .|\operatorname{Im} p|<\pi / 4\}$, supp $g_{2} \subset$ $\{p: 0<|\operatorname{Im} p|<\pi / 2\}, g_{3}$ being $i \pi-$ periodic function. Then

$$
\begin{gathered}
E_{\lambda}^{\alpha} f(0)=I_{1 \delta}(\lambda)+I_{2 \delta}(\lambda)+I_{3 \delta}(\lambda), \\
I_{\jmath, \delta}(\lambda)=\lambda^{-\alpha} \Gamma(\alpha+1)(2 \pi i)^{-1} \int_{S} e^{\lambda p} H_{\alpha}(\lambda+n, p) u(p, 0) g_{\jmath} d p,
\end{gathered}
$$

where $S_{1}=S_{2}=S, S_{3}=(\delta+i 0, \delta+i \pi), g_{\jmath} \in C_{0}^{\infty}\left(S_{\jmath}\right), j=1,2,3$. In obtaining the third integral we have used the periodicity of the integrand in (6.4). Since

$$
u(p, 0)=c_{\beta}\left(p^{-1} \sinh 2 p\right)^{\beta / 2}(\cosh 2 p)^{-\beta / 2-n / 2} p^{\beta / 2}
$$

we have

$$
I_{1 \delta}(\lambda)=\lambda^{-\alpha}\left(\int_{S_{1}} e^{\lambda p} p^{-\alpha-1+\beta / 2} q_{1}(p) d p+\int_{S_{1}} e^{\lambda p} p^{\beta / 2} q_{2}(p) d p\right),
$$

where $q_{\jmath} \in C_{0}^{\infty}\left(S_{1}\right)$.

On the other hand, we obtain

$$
I_{2 \delta}(\lambda)=\lambda^{n / 2+\beta / 2-\alpha} \int e^{\lambda \phi(p, \sigma)} q(p, \sigma) d p d \sigma+e^{\lambda \delta} O\left(\lambda^{-\infty}\right)
$$

where $q \in C_{0}^{\infty}\left(S_{2} \times(0, \infty)\right), \phi(p, \sigma)=p-2^{-1} \sigma^{2} \operatorname{coth} 2 p$. Here we have integrated by parts and used the bound $\left|\partial_{p} \phi\right| \geq c>0$ for $\sigma \sim 0$ or $\sigma \sim \infty$. Consequently (6.5)-(6.7) give

$$
E_{\lambda}^{\alpha} f(0)=I(\lambda)+O\left(\lambda^{-\beta / 2}\right)
$$

where

$$
\begin{gathered}
I(\lambda)=\lambda^{n / 2+\beta / 2-\alpha} \int e^{2 \lambda \phi(t, \sigma)} q(t, \sigma) d t d \sigma, \phi(t, \sigma)=t+2^{-1} \sigma^{2} \cot 2 t, \\
q(t, \sigma)=2^{-n / 2} \pi^{-3 / 2} / \Gamma(n / 2-1 / 2) H_{\alpha}(\lambda+n, i t) g_{2}(i t)(i \sin 2 t)^{-n / 2} \sigma^{n-1+\beta} g(\sigma),
\end{gathered}
$$

and $g \in C_{0}^{\infty}(0, \infty)$.

Now the method of the stationary phase implies

$$
I(\lambda)=\lambda^{n / 2+\beta / 2-\alpha-1}\left(a(\lambda)+O\left(\lambda^{-1}\right)\right) .
$$

Evidently (6.1) follows from (6.8), (6.9).

\section{Proof of theorem 4}

Starting with the formula (6.2) and having in mind the singularities at the points $p=0, p=$ $\pm i \pi / 2$, we write

$$
e_{\alpha}(\lambda, x, y)=\sum_{\jmath=1}^{3} e_{j}(\lambda, x, y, \delta)
$$

where

$$
e_{j}(\lambda, x, y, \delta)=b \int_{S} e^{\lambda p} V(p, x, y) H_{\alpha}(\lambda+n, p) g_{\jmath}(p) d p
$$

$g_{\text {j }}$ are $C^{\infty}$ functions, $g_{1}(p)+g_{2}(p)+g_{3}(p)=1$ for $p \in S$, supp $g_{1} \subset\{p \in S:|\operatorname{Im} p|<\epsilon\}$, supp $g_{2} \subset$ $\{p \in S:|\operatorname{Im} p|>\pi / 2-\epsilon\}$ for some small $\epsilon>0$, and $g_{2}$ is $i \pi-$ periodic. Here $b=\Gamma(\alpha+1)(2 \pi i)^{-1}$.

If $j=1$ we shall use the representations:

$$
V(p, x, y)=a(p, x, y) \int_{\mathbf{R}^{n}} \exp \left(-\xi^{2} p+i(x-y) \xi\right) d \xi(2 \pi)^{-n}, \operatorname{Re} p>0,
$$

where $a(0, x, y)=1, p \rightarrow a(p, x, y)$ is smooth for $p \in S$ and

$$
\int_{\mathbf{R}^{2}} e^{-p \eta^{2}} \eta^{2 \alpha} d \eta=\pi \Gamma(\alpha+1) p^{-\alpha-1}, \operatorname{Re} p>0
$$


Since

$$
H_{\alpha}(\lambda+n, p)=p^{-\alpha-1}+h_{\alpha}(\lambda+n, p)
$$

and $h_{\alpha}$ has no singularities on $S$, we have

$$
e_{1}(\lambda, x, y, \delta)=\lambda^{n / 2+\alpha+1} I_{1}+\lambda^{n / 2} I_{2},
$$

where

$$
\begin{gathered}
I_{1}=-i c_{1} \int_{S \times \mathbf{R}^{n} \times \mathbf{R}^{2}} e^{\lambda\left(1-\xi^{2}-\eta^{2}\right) p+\imath \sqrt{\lambda}(x-y) \xi} a(p, x, y) g_{1}(p) \eta^{2 \alpha} d p d \xi d \eta, \\
I_{2}=c_{2} \int_{S \times \mathbf{R}^{n}} e^{\lambda\left(1-\xi^{2}\right) p+\imath \sqrt{\lambda}(x-y) \xi} a(p, x, y) h_{\alpha}(\lambda+n, p) g_{1}(p) d p d \xi,
\end{gathered}
$$

$c_{1}=(2 \pi)^{-n-1} \pi^{-1}$ and $c_{2}$ is a constant.

In both integrals $I_{1}, I_{2}$ we can suppose that the integration with respect to $(\xi, \eta)$ or $\xi$ is taken over a ball, the rest being estimated with $O\left(e^{c \delta \lambda} \lambda^{-\infty}\right), c>0$.

To represent $e_{2}$ we first use $i \pi-$ periodicity of the integrand in (7.2) and conclude that we can suppose $g_{2} \in C_{0}^{\infty}$, supp $g_{2} \subset\{p=\delta+i t:|t-\pi / 2|<\epsilon\}$. The translation $p \rightarrow p+i \pi / 2$ finally gives

$$
e_{2}(\lambda, x, y, \delta)=b \int_{\delta+\imath \mathbf{R}} e^{\lambda(p+\imath \pi / 2)} V(p+i \pi / 2, x, y) H_{\alpha}(\lambda+n, p+i \pi / 2) g_{2}(p) d p
$$

where $g_{2} \in C_{0}^{\infty}$, supp $g_{2} \subset\{p=\delta+i t:|t|<\epsilon\}$.

According to (6.3)

$$
V(p+i \pi / 2, x, y)=(-2 \pi \sinh 2 p)^{-n / 2} \exp \left(-\frac{x^{2}+y^{2}}{2} \operatorname{coth} 2 p-\frac{x y}{\sinh 2 p}\right)
$$

whence

$$
V(p+i \pi / 2, x, y)=b(p, x, y) \int_{\mathbf{R}^{n}} \exp \left(-\xi^{2} p+i(x+y) \xi\right) d \xi, \operatorname{Re} p>0
$$

and $b(0, x, y)=(2 \pi)^{-n / 2} e^{-\imath \pi n / 2}$. Thus

$$
\begin{gathered}
e_{2}(\lambda, x, y, \delta)=\lambda^{n / 2} \int_{S \times \mathbf{R}^{n}} e^{\lambda\left(1-\xi^{2}\right) p+\imath \sqrt{\lambda}(x+y) \xi} q_{2}(\lambda, p, x, y) d p d \xi \\
q_{2}=c_{3} b(p, x, y) H_{\alpha}(\lambda+n, p+i \pi / 2) g_{2}(p) e^{i \lambda \pi / 2}
\end{gathered}
$$

for some constant $c_{3}$.

Analogously,

$$
e_{3}(\lambda, x, y, \delta)=\lambda^{n / 2} \int_{S \times \mathbf{R}^{n}} e^{\lambda\left(1-\xi^{2}\right) p+i \sqrt{\lambda}(x-y) \xi} q_{3}(\lambda, p, x, y) d p d \xi
$$

$q_{3}=c_{4} a(p, x, y) H_{\alpha}(\lambda+n, p) g_{3}(p)$.

Since the functions $p \rightarrow q_{j}$ are $C_{0}^{\infty}$ we can integrate by parts in the integrals $e_{j}, j=2,3$. So the integration with respect to $\xi$ is over a ball, the rest being estimated with $O\left(e^{-c \delta \lambda} \lambda^{-\infty}\right), c>0$. Now letting $\delta \rightarrow 0$ in (7.1), (7.4)-(7.8) we obtain

$$
\begin{gathered}
e_{\alpha}(\lambda, x, y)=\sum_{j=1}^{3} e_{j}(\lambda, x, y)+O\left(\lambda^{-\infty}\right) \\
e_{1}=\lambda^{n / 2+\alpha+1} I_{1}+\lambda^{n / 2} I_{2} \\
I_{1}=\int_{\mathbf{R} \times \mathbf{R}^{n} \times \mathbf{R}^{2}} e^{i \lambda \phi_{1}} q_{1} d t d \xi d \eta \\
I_{2}=\int_{\mathbf{R} \times \mathbf{R}^{n}} e^{\imath \lambda \phi_{2}} q_{2} d t d \xi
\end{gathered}
$$

where $\phi_{1}=\left(1-\xi^{2}-\eta^{2}\right) t+\lambda^{-1 / 2}(x-y) \xi, \phi_{2}=\left(1-\xi^{2}\right) t+\lambda^{-1 / 2}(x-y) \xi$ and $q_{1}=c_{1} a(i t, x, y) g_{1}(i t) g(\xi, \eta)$, $g$ being a cutoff function, and $q_{2} \in C_{0}^{\infty}$. 
Notice that $e_{j}(\lambda, x, y), j=2,3$ have the same form as $\lambda^{n / 2} I_{2}(\lambda, x, y)$, therefore it suffices to find the asymptotics of the integrals $I_{\jmath}, j=1,2$.

To find the asymptotics of $I_{1}$ we use polar coordinates $(\xi, \eta)=\sigma(\omega, \theta), \omega \in \mathbf{R}^{n}, \sigma>0, \omega^{2}+$ $\theta^{2}=1$ and the equality

$$
\int_{\omega^{2}+\theta^{2}=1} e^{2 \sqrt{\lambda}(x-y) \omega \sigma} \theta^{2 \alpha} d(\omega, \theta)=c(\sqrt{\lambda}|x-y| \sigma)^{-n / 2-\alpha} J_{n / 2+\alpha}(\sqrt{\lambda}|x-y| \sigma),
$$

$c=(2 \pi)^{n / 2} 2^{\alpha+1} \pi \Gamma(\alpha+1)$. Therefore

$$
I_{1}=(\sqrt{\lambda}|x-y|)^{-n / 2-\alpha} \int_{\mathbf{R}} \int_{0}^{\infty} e^{2 \lambda\left(1-\sigma^{2}\right) t} \sigma^{n / 2+\alpha+1} J_{n / 2+\alpha}(\sqrt{\lambda}|x-y| \sigma) q d t d \sigma
$$

where

$$
t \rightarrow q \in C_{0}^{\infty} \text { and } q(0,1)=(2 \pi)^{-n / 2-1} 2^{\alpha+1} \Gamma(\alpha+1) .
$$

Integrating by parts in the integral (7.13) with respect to $t$ when $\sigma$ is close to zero, we can suppose that $q(t, \sigma)$ has a compact support in $\mathbf{R} \times(0, \infty)$, the rest being estimated with $O\left(\lambda^{-\infty}\right)$.

Let $\sqrt{\lambda}|x-y|>1$. Then we shall use the formula [20, p. 168].

$$
J_{n / 2+\alpha}(r)=r^{-1 / 2}\left(e^{\imath r} f(r)+e^{-\imath r} \bar{f}(r)\right), r>0
$$

and the bound

$$
\left|\partial_{\sigma}^{k} f(\sqrt{\lambda}|x-y| \sigma)\right| \leq c_{k} \text { if } \sqrt{\lambda}|x-y|>1,0<a \leq \sigma \leq b .
$$

Consider the phase function $\psi$ :

$$
\psi(t, \sigma, x, y)=\left(1-\sigma^{2}\right) t \pm|x-y| \lambda^{-1 / 2} \sigma .
$$

The critical points $(t, 1)$ are nondegenerate and

$$
q(t, 1)=q(0,1)+|x-y| O\left(\lambda^{-1 / 2}\right) .
$$

Hence the method of the stationary phase implies for $\sqrt{\lambda}|x-y|>1$ :

$$
I_{1}=(\sqrt{\lambda}|x-y|)^{-n / 2-\alpha}\left(d_{\alpha} \lambda^{-1} J_{n / 2+\alpha}(\sqrt{\lambda}|x-y|)+|x-y|^{-1 / 2} O\left(\lambda^{-7 / 4}\right)\right),
$$

where $d_{\alpha}=(2 \pi)^{-n / 2} 2^{\alpha} \Gamma(\alpha+1)$.

Consider now the case $\sqrt{\lambda}|x-y|<1$. Then we can write

$$
\begin{gathered}
I_{1}=\int_{\mathbf{R}} \int_{0}^{\infty} e^{2 \lambda\left(1-\sigma^{2}\right) t} g(t, \sigma, \lambda) d t d \sigma, \\
g(t, \sigma, \lambda)=c_{1} \int_{\omega^{2}+\theta^{2}=1} e^{\imath \sqrt{\lambda}(x-y) \omega \sigma} \theta^{2 \alpha} d(\omega, \theta) \sigma^{n+2 \alpha+1} q_{1}(t, \sigma) .
\end{gathered}
$$

The method of the stationary phase shows that

$$
I_{1}=\pi c_{1} \lambda^{-1} \int_{\omega^{2}+\theta^{2}=1} e^{2 \sqrt{\lambda}(x-y) \omega} \theta^{2 \alpha} d(\omega, \theta)+O\left(\lambda^{-2}\right),
$$

whence

$$
I_{1}=d_{\alpha}(\sqrt{\lambda}|x-y|)^{-n / 2-\alpha} J_{n / 2+\alpha}(\sqrt{\lambda}|x-y|) \lambda^{-1}+O\left(\lambda^{-2}\right)
$$

if $\sqrt{\lambda}|x-y|<1$.

On the other hand, in polar coordinates $\xi=\sigma \omega$,

$$
I_{2}=\int_{\mathbf{R} \times(0, \infty) \times S^{n-1}} e^{\imath \lambda\left(1-\sigma^{2}\right) t+\imath \sqrt{\lambda}(x-y) \omega \sigma} \sigma^{n-1} q_{2}(t, \sigma) d t d \sigma d \omega,
$$

whence the stationary phase method gives

$$
I_{2}=O\left(\lambda^{-1}\right)
$$

Thus (2.2), (7.9), (7.10), (7.18)-(7.20) imply (2.3) for $\alpha \geq(n-1) / 2$. Theorem 4 is proved. 


\section{Proof of theorem 5}

Starting with (6.2),(6.3) we see that the phase function $p \rightarrow \phi(p, x, y)$, given by

$$
\phi(p, x, y)=p-2^{-1}\left(x^{2}+y^{2}\right) \operatorname{coth} 2 p+x y(\sinh 2 p)^{-1},
$$

has the critical points $p_{ \pm}=i t_{ \pm}$and $\bar{p}_{ \pm}$, where $\cos 2 t_{ \pm}=x y \mp d$ and $d^{2}=(x y)^{2}+1-x^{2}-y^{2}$. If $x=y$ then $p_{-}=0$ and the integrand in (6.2) is not holomorphic function in a neighborhood of the critical points. So we have to expand the singularities. Analogously to (7.1), (7.2) we can write

$$
E_{\alpha}(\lambda, x, y)=\sum_{j=1}^{3} E_{j}(\lambda, x, y, \delta)
$$

where

$$
E_{\jmath}=(2 \pi i)^{-1} \Gamma(\alpha+1) \int_{S} e^{\lambda p} V(p, \sqrt{\lambda} x, \sqrt{\lambda} y) H_{\alpha}(\lambda+n, p) g_{j}(p) d p
$$

Further,

$$
V(p, x, y)=(2 \pi)^{-n} \int_{\mathbf{R}^{n}} \exp \left(-\frac{x^{2}+y^{2}}{2} \tanh p-\frac{\xi^{2}}{2} \sinh 2 p+i(x-y) \xi\right) d \xi,
$$

and for $\operatorname{Re} p>0$,

$$
1=c_{\alpha}(\sinh 2 p)^{\alpha+1} \int_{\mathbf{R}^{2}} \eta^{2 \alpha} \exp \left(-\frac{1}{2} \eta^{2} \sinh 2 p\right) d \eta .
$$

Analogously to (7.3) we have

$$
E_{1}(\lambda, x, y, \delta) \sim \lambda^{n / 2+\alpha+1} I_{1}(\lambda, x, y, \delta)+I_{2}(\lambda, x, y, \delta),
$$

where now

$$
\begin{gathered}
I_{1}=\int_{\mathbf{R}^{n} \times \mathbf{R}^{2} \times S} e^{\lambda \phi_{1}} q_{1} d \xi d \eta d p, \\
\phi_{1}=p-2^{-1}\left(\xi^{2}+\eta^{2}\right) \sinh 2 p-2^{-1}\left(x^{2}+y^{2}\right) \tanh p+i(x-y) \xi,
\end{gathered}
$$

$q_{1}=(p / \sinh 2 p)^{-\alpha-1} g(\xi, \eta) \eta^{2 \alpha} g_{1}(p)$ and

$$
\begin{gathered}
I_{2}=\lambda^{n / 2} \int_{\mathbf{R}^{n} \times S} e^{\lambda \phi_{2}} q_{2} d \xi d p \\
\phi_{2}=p-2^{-1} \xi^{2} \sinh 2 p-2^{-1}\left(x^{2}+y^{2}\right) \tanh p+i(x-y) \xi,
\end{gathered}
$$

$q_{2}=h_{\alpha}(\lambda, p) g(\xi) g_{1}(p)$ for some cutoff function $g$.

To represent $E_{2}$ we use the periodicity of the integrand in (6.2) and the formula

$$
V\left(p+i \frac{\pi}{2}, x, y\right)=a(p) \int_{\mathbf{R}^{n}} \exp \left(-\frac{\xi^{2}}{2} \tanh 2 p+x y \tanh +x y \tanh p+i(x+y) \xi\right) d \xi
$$

where $a(p)=\left(4 \pi^{2} \cosh 2 p\right)^{-n / 2}$. Thus

$$
\begin{gathered}
E_{2}(\lambda, x, y, \delta) \sim \lambda^{n / 2} \int_{\mathbf{R}^{n} \times S} e^{\lambda \psi} q d \xi d p, \\
\psi=p-2^{-1} \xi^{2} \tanh 2 p+x y \tanh p+i(x+y) \xi, \\
q=(\cosh 2 p)^{-n / 2} H_{\alpha}(\lambda+n, p+i \pi / 2) g(\xi) g_{2}(p+i \pi / 2) e^{2 \lambda \pi / 2} .
\end{gathered}
$$

Therefore letting $\delta \rightarrow 0$ we obtain from (8.3)-(8.6)

$$
E_{\alpha}(\lambda, x, y)=\sum E_{j}(\lambda, x, y)+O\left(\lambda^{-\infty}\right)
$$

where

$$
E_{1}=\lambda^{n / 2+\alpha+1} I_{1}+\lambda^{n / 2} I_{2}
$$


the integrals $I_{3}$ being given by (7.11), (7.12), but now

$$
\begin{gathered}
\phi_{1}=t-2^{-1}\left(\xi^{2}+\eta^{2}\right) \sin 2 t-2^{-1}\left(x^{2}+y^{2}\right) \tan t+(x-y) \xi, \\
\phi_{2}=t-2^{-1} \xi^{2} \sin 2 t-2^{-1}\left(x^{2}+y^{2}\right) \tan t+(x-y) \xi, \\
q_{1}=(t / \sin 2 t)^{-\alpha-1} g(\xi, \eta) \eta^{2 \alpha} g_{1}(i t), q_{2}=(\cos 2 t)^{-n / 2} h_{\alpha}(\lambda, i t) g(\xi) g_{1}(i t) .
\end{gathered}
$$

Further, $E_{2}$ is analogous to $I_{2}$ and

$$
\begin{gathered}
E_{3}=\int_{\mathbf{R}} e^{\imath \lambda \phi_{3}} q_{3} d t, \phi_{3}=t+2^{-1}\left(x^{2}+y^{2}\right) \cot 2 t-x y(\sin 2 t)^{-1}, \\
q_{3}=(2 \pi)^{-1} \Gamma(\alpha+1)(2 \pi i \sin 2 t)^{-n / 2} H_{\alpha}(\lambda+n, i t) g_{3}(i t) .
\end{gathered}
$$

To find the uniform asymptotics of the integrals $E_{\jmath}$ in the domain $\left\{x, y \in \mathbf{R}^{n}: A / \lambda<x^{2}<\right.$ $1-\delta,|y|<\epsilon|x|\}$ we shall apply the method of the stationary phase.

a. Asymptotics of $I_{1}$. Analogously to (7.13) we have

$$
I_{1}=(\lambda|x-y|)^{-n / 2-\alpha} \int_{\mathbf{R}} \int_{0}^{\infty} e^{\imath \lambda \psi_{s}} \sigma^{n / 2+\alpha+1} J_{n / 2+\alpha}(\lambda|x-y| \sigma) q(t, \sigma) d t d \sigma,
$$

where $\psi_{0}=t-2^{-1} \sigma^{2} \sin 2 t-2^{-1}\left(x^{2}+y^{2}\right) \tan t, q \in C_{0}^{\infty}(\mathbf{R} \times(0, \infty))$. Here we have integrated by parts using the estimate $\left|\partial_{t} \psi_{o}\right| \geq c>0$ if $\sigma$ is close to zero.

Since $|x-y|>c|x|>c \lambda^{-1 / 2}$ we have for $s=\lambda|x-y| \sigma$

$$
J_{n / 2+\alpha}(s)=\sum_{k=0}^{2} s^{-1 / 2-k} c_{k} \cos \left(s+b_{k}\right)+|x|^{-1 / 2} O\left(\lambda^{-2}\right),
$$

where $b_{k}$ is a constant. Therefore

$$
I_{1}=\sum_{k=0}^{2}(\lambda|x-y|)^{-(n+1) / 2-\alpha-k} M_{k}+(\lambda|x|)^{-(n+1) / 2-\alpha} O\left(\lambda^{-3 / 2}\right),
$$

where

$$
\begin{aligned}
& M_{k}=\int_{\mathbf{R}} \int_{0}^{\infty} e^{2 \lambda \psi} \sigma^{(n+1) / 2+\alpha-k} q_{k}(t, \sigma) d t d \sigma, q_{k} \in C_{0}^{\infty}, \\
& \psi=t-2^{-1} \sigma^{2} \sin 2 t-2^{-1}\left(x^{2}+y^{2}\right) \tan t \pm|x-y| \sigma .
\end{aligned}
$$

The critical points $\left(t_{\jmath}, \sigma_{\jmath}\right)$ of $\psi$ satisfy

$$
\cos 2 t_{j}=x y+(-1)^{j+1} d(j=1,2), t_{3}=-t_{1}, t_{4}=-t_{2}, \sigma_{j} \sin 2 t_{j}= \pm|x-y|,
$$

where $d^{2}=(x y)^{2}+1-x^{2}-y^{2}$. Since $x^{2}<1-\delta,|y|<\epsilon|x|$ for small $\delta>0, \epsilon>0$ and the support of $t \rightarrow q_{k}(t, \sigma)$ is small enough, we have $d>c>0$, $\operatorname{det} \psi^{\prime \prime}= \pm 4 d$ for the Hessian $\psi^{\prime \prime}$ in the critical points. Therefore the critical points are nondegenerate. Thus the stationary phase method implies

$$
I_{1}=\lambda^{-(n+1) / 2-\alpha-1} \sum_{j=1}^{4} e^{2 \lambda \psi} b_{\jmath} g_{1}\left(i t_{\jmath}\right)+(\lambda|x|)^{-(n+1) / 2-\alpha} O\left(\lambda^{-3 / 2}\right)
$$

and $b_{\jmath}, \psi_{\jmath}$ have the properties $(2.4),(2.5)$ respectively.

b. Asymptotics of $I_{2}$. In polar coordinates $\xi=\sigma \omega, \sigma>0$ we have

$$
\begin{gathered}
I_{2}=\int_{\mathbf{R}} \int_{0}^{\infty} \int_{|\omega|=1} e^{\imath \lambda \psi} q(t, \sigma) d t d \sigma d \omega, q \in C_{0}^{\infty}, \\
\psi=t-2^{-1} \sigma^{2} \sin 2 t-2^{-1}\left(x^{2}+y^{2}\right) \tan t+(x-y) \omega \sigma .
\end{gathered}
$$

Since the support of $t \rightarrow q(t, \sigma)$ is small, the critical points $(t, \sigma)$ of $\psi$ are nondegenerate if $x^{2}<1-\delta,|y|<\epsilon|x|$ for small $\delta>0, \epsilon>0$. Hence for large $M$,

$$
I_{2}=\sum \lambda^{-k} \int_{|\omega|=1} e^{\imath \lambda \phi_{\jmath}} a_{k \jmath}(\lambda, x, y, \omega) d \omega+O\left(\lambda^{-M-1}\right)
$$


where $1 \leq k \leq M, 1 \leq j \leq 4$ and $\phi_{\jmath}=(x-y) \omega a_{\jmath}(x, y, \omega), a_{\jmath}(x, x, \omega)=\left(1-x^{2}\right)^{1 / 2}$.

Since $\lambda|x-y|>c \sqrt{\lambda}$ the method of the stationary phase gives

$$
I_{2}=\lambda^{-(n+1) / 2} \sum_{\jmath=1}^{4} e^{2 \lambda \psi_{j}} b_{\jmath} g_{1}\left(i t_{\jmath}\right)+|x|^{-(n+1) / 2} O\left(\lambda^{-(n+3) / 2}\right) .
$$

Notice that $E_{2}$ has the same asymptotics (8.10), where $g_{1}$ is replaced by $g_{2}$.

c. Asymptotics of $E_{3}$. The critical points $t_{3}$ of the phase function $\phi_{3}$ satisfy $\cos 2 t_{0}=x y+$ $(-1)^{\jmath+1} d$ and $\phi_{3}^{\prime \prime}\left(t_{\jmath}, x, y\right)=(-1)^{\jmath+1} 4 d\left(\sin 2 t_{\jmath}\right)^{-1}, 1 \leq j \leq 4$. Therefore the stationary phase method implies

$$
E_{3}=\lambda^{-1 / 2} \sum_{\jmath=1}^{4} b_{\jmath} e^{2 \lambda \psi_{\jmath}} g_{3}\left(i t_{\jmath}\right)+O\left(\lambda^{-3 / 2}\right)
$$

Evidently, theorem 5 follows from (8.7)-(8.11).

\section{Proof of theorem 6}

Starting with (6.2), (2.2), we can write

$$
E_{\alpha}(\lambda, x, y)=\int_{S} e^{\lambda \phi} q d p
$$

where the function $\phi$ is given by (8.1) and

$$
q(p)=\Gamma(\alpha+1)(2 \pi i)^{-1}(2 \pi \sinh 2 p)^{-n / 2} H_{\alpha}(\lambda+n, p) .
$$

Now the problem is to find the uniform asymptotics of the integral (9.1) as $\lambda \rightarrow \infty$. The critical points of the phase function $p \rightarrow \phi(p, x, y)$ satisfy the relation $\cosh 2 p=x y+d$, where $d^{2}=$ $(x y)^{2}+1-x^{2}-y^{2}$. Let $x=r \omega,|\omega|=1$. Then the critical points degenerate if $r=a$, where $a=a(y, \omega)$ is given by $(2.7)$. We have two degenerate critical points: $p_{0}$ and $\bar{p}_{0}$, where $p_{0}=i t_{0}$ and $\cos 2 t_{0}=a \omega y, t_{0}>0$. In particular, $t_{0}<\pi / 2$ if $|y|$ is sufficiently small. Thus if $\left|x^{2}-1\right|<$ $\delta,|y|<\epsilon|x|$ for some small $\delta>0, \epsilon>0$ there are only four critical points $p_{ \pm}, \bar{p}_{ \pm}$, where

$$
\begin{gathered}
p_{ \pm}=i t_{ \pm}, \cos 2 t_{ \pm}=x y \pm d, 0<t_{ \pm}<\pi / 2 \text { if } x^{2}<a^{2} \\
p_{ \pm}= \pm \delta+i t, \cosh 2 \delta \cos 2 t=x y, 0<t<\pi / 2 \text { if } x^{2}>a^{2},
\end{gathered}
$$

and $2 \cos ^{2} 2 t=x^{2}+y^{2}-\left(\left(x^{2}+y^{2}\right)^{2}-4(x y)^{2}\right)^{1 / 2}$.

Near these critical points the integrand in (9.1) is a holomorphic function and $\partial^{3} \phi / \partial p^{3}=$ $b_{1}(y, \omega), \partial^{2} \phi / \partial p \partial r=-b_{2}(y, \omega)$ for $p=p_{0}$ or $p=\bar{p}_{0}$, where $b_{1}(o, \omega)=8, b_{2}(0, \omega)=2$. Therefore we can apply Lemma 2.3 in [5], p.343 and conclude that there exists a holomorphic change of variables $p=p(z, x, y)$, defined in a neighborhood of the points $z=0, r=a$ such that

$$
\phi(p(z, x, y), x, y)=A(x, y)-B(x, y) z+z^{3} / 3, p(0, a w, y)=p_{0},
$$

for every fixed $\omega, y$. In addition, the coefficients $A, B$ are given by

$$
\begin{gathered}
\left.A=\frac{1}{2}\left(\phi\left(p_{+}, x, y\right)+\phi_{-}, x, y\right)\right), \\
B=\left(\frac{3}{4}\left(\phi\left(p_{+}, x, y\right)-\phi\left(p_{-}, x, y\right)\right)\right)^{2 / 3},
\end{gathered}
$$

and $p( \pm \sqrt{B}, x, y)=p_{ \pm}$.

To use this change of variables in the integral (9.1), we notice first that

$$
E_{\alpha}(\lambda, x, y) \sim \int_{L} e^{\lambda \phi} q d p, L=L_{1} \cup L_{2},
$$


where $L_{1}$ is the segment $\left(\delta+i\left(t_{0}-2 \delta, \delta+i\left(t_{0}+2 \delta\right)\right)\right.$ and $L_{2}$ - the segment $\left(\delta-i\left(t_{0}+2 \delta\right), \delta+i\left(-t_{0}+2 \delta\right)\right)$ for $\delta>0$ small enough. The equivalence relation " $a(\lambda, x, y) \sim b(\lambda, x, y)$ " here means that $a-b=$ $O\left(e^{-c \lambda}\right), c>0$. Indeed, it is sufficient to notice the bound $\operatorname{Re} \phi(p, x, y) \leq-c<0$ for $p \in S \backslash L$, which follows from the definition (8.1) if $\delta>0$ is small enough.

Now (9.1)-(9.3) yield

$$
E_{\alpha}(\lambda, x, y) \sim \sum_{\jmath=1}^{2} e^{\lambda A_{\jmath}} \int_{L_{\jmath}} e^{\lambda\left(-B z+z^{3} / 3\right)} q_{\jmath}(z, \lambda) d z
$$

where $A_{1}=A, A_{2}=\bar{A}, q_{1}(z, \lambda)=q(p(z, x, y)) \partial p / \partial z, q_{2}(z, \lambda)=q(\bar{p}(\bar{z}, x, y)) \times \partial \bar{p} / \partial z, L_{1}$, being the image of the segment $L_{\jmath}$. Notice that $L_{1 j} \subset\{z: \operatorname{Re} z>0\}$ and that the end points $a_{\jmath}, b_{\jmath}$ of $L$, satisfy $\arg a_{\jmath} \in(-\pi / 2,-\pi / 6)$, $\arg b_{\jmath} \in(\pi / 6, \pi / 2)$.

Using the Weierstrass preparation theorem [9]:

$$
q_{\jmath}(z, \lambda)=r_{\jmath}+r_{1 \jmath} z+\left(z^{2}-B\right) q_{1 \jmath}(z, \lambda)
$$

and the following representation of the Airy function

$$
A i(s)=(2 \pi i)^{-1} \int_{M} e^{-s z+z^{3} / 3} d z, M=M_{1} \cup M_{2},
$$

$M_{1}: z=r e^{\imath \theta}, r \in(+\infty, 0), \theta \in(-\pi / 2,-\pi / 6), M_{2}: z=r e^{\imath \theta}, r \in(0,+\infty), \theta \in(\pi / 6, \pi / 2)$, in the integral (9.4), we obtain the uniform asymptotics (2.6), the rest being estimated as in [5], p. 348.

\section{Proof of theorem 7}

Now we use the formula (6.2) with $\delta=\delta(x, y)>0$ such that $2 \cosh ^{2} 2 \delta=x^{2}+y^{2}+$ $\left(\left(x^{2}+y^{2}\right)^{2}-4(x y)^{2}\right)^{1 / 2}$. The critical points $p(x, y)=\delta+i t$ and $\bar{p}(x, y)$ are nondegenerate and $\operatorname{Re} \phi(p, x, y)<\operatorname{Re} \phi(p(x, y), x, y)$ if $0 \leq \operatorname{Im} p \leq \pi / 2, p \neq p(x, y) ; \operatorname{Re} \phi(p, x, y)<\operatorname{Re} \phi(\bar{p}(x, y), x, y)$ if $-\pi / 2 \leq \operatorname{Im} p \leq 0, p \neq \bar{p}(x, y)$. In addition, $\partial^{2} \phi / \partial p^{2}(p(x, y), x, y)=4 d / \sinh 2 p(x, y)$ and $\operatorname{Re} \phi(p(x, y), x, y)=2^{-1}\left(\operatorname{arcosh} \beta-\beta \sqrt{\beta^{2}-1}\right), \beta=\cosh 2 \delta$. Since $\beta^{2}-1 \geq c\left(x^{2}-1\right), c>0$ and $\beta \sqrt{\beta^{2}-1}-\operatorname{arcosh} \beta \geq \gamma \sqrt{\beta^{2}-1}$ if $\beta^{2}-1>\gamma$, for some $0<\gamma<1$, one obtains theorem 7 by the saddle-point method.

Acknowledgement. The authors would like to thank the referee for various suggestions which improved the paper.

\section{REFERENCES}

[1] ASKEY, R. and WAINGER, S., Means convergence of expansions in Laguerre and Hermite series, Amer. J. Math., 87 (1965), 695-708.

[2] BERARD, P. H., Riesz means on Riemannian manifolds, Proc. Symp. Pure Math., 36 (1980), 1-12.

[3] CHRIST, F. M., C. D. Sogge, The weak type $L^{1}$ convergence of eigenfunction expansions for pseudodifferential operators, Invent. Math., 94 (1988), 421-453.

[4] COLZANI, L. and TRAVAGLINI, G., Estimates for Riesz kernels of eigenfunction expansions of elliptic differential operators on compact manifolds, J. Funct. Anal., 96 (1991), 1-30.

[5] FEDORJUK, M., Method Perevala, Nauka, Moscow, 1977. 
[6] GURARIE, D., Kernels of elliptic operators: bounds and summability, J. Differentıal Equations, 55 (1984), 1-29.

[7] HÖRMANDER, L., On the Riesz means of spectral functions and eigenfunction expansions for elliptic differential operators, in Some Recent Advances in the Basıc Scrences, 155-202. Yeshiva University, New York, 1966.

[8] HÖRMANDER, L., The spectral function of an elliptic operator, Acta Math., 121 (1968), 193-218.

[9] HÖRMANDER, L., The Analysis of Linear Partial Differential Operators I. Springer, New York, 1983.

[10] KARADZHOV, G. E., Equiconvergence theorems for Laguerre series, Banach Center Publ. 27 (1992), 207-220.

[11] KON, M., RAPHAEL, L. and YOUNG, J., Kernels and equisummation properties of uniformly elliptic operators, J. Differential Equations, 67 (1987), 256-268.

[12] KON, M., Summation and spectral theory of eigenfunction expansions, in Discourses in Math. and its applications, 1 (1991), Dept. of Math., Texas AM University, College Station, 49-76.

[13] MUCKENHOUPT, B., Mean convergence of Hermite and Laguerre series, Trans. Amer. Math. Soc., 147 (1970), 419-460.

[14] POIANI, E. L., Mean Cesaro summability of Laguerre and Hermite series, Trans. Amer. Math. Soc., 173 (1972), 1-31.

[15] SOGGE, C. D., Fourier integrals in classical analysis, Cambridge Tracts in Math., 105, Cambridge Univ. Press, 1993.

[16] STEIN, E. M. and WEISS, G., Introduction to Fourier Analysis on Euclidean Spaces, Princeton Univ. Press, 1971.

[17] STRICHARTZ, R., Harmonic analysis as spectral theory of Laplacians, J. Funct. Anal., 87 (1989), 51-148.

[18] SZEGÖ, G., Orthogonal polynomials, Amer. Math. Soc. Colloq. Publ., Vol. 23, Amer. Math. Soc., Providence, RI, 1959.

[19] THANGAVELU, S. , Lectures on Hermite and Laguerre expansions, Math. Notes, 42, Princeton Univ. Press, 1993.

[20] WATSON, G., A Treatise on the Theory of Bessel Functions, Cambridge Univ. Press, 1966. 


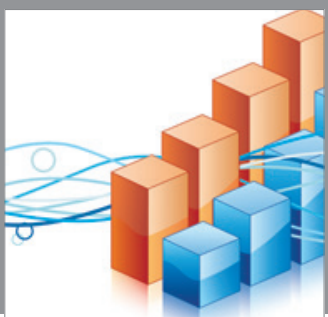

Advances in

Operations Research

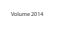

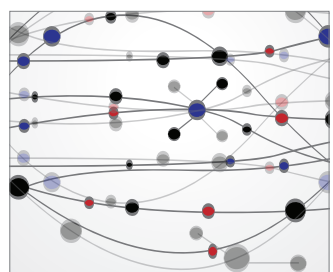

\section{The Scientific} World Journal
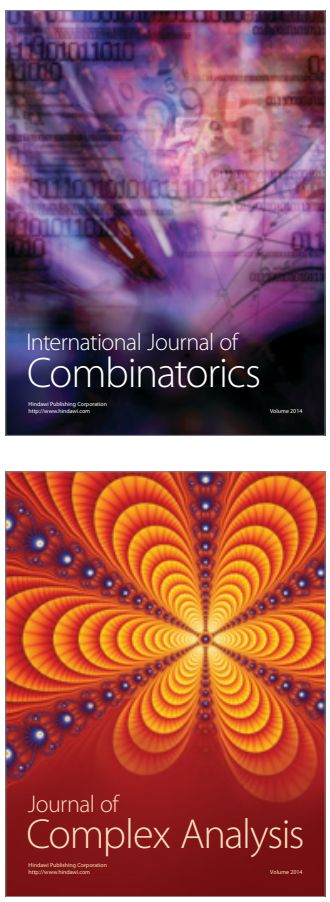

International Journal of

Mathematics and

Mathematical

Sciences
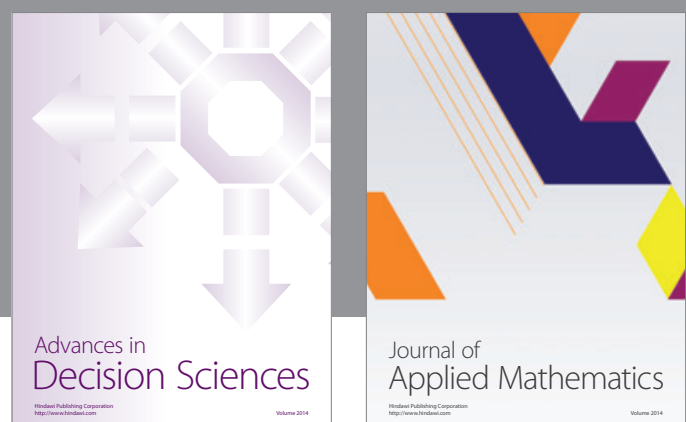

Journal of

Applied Mathematics
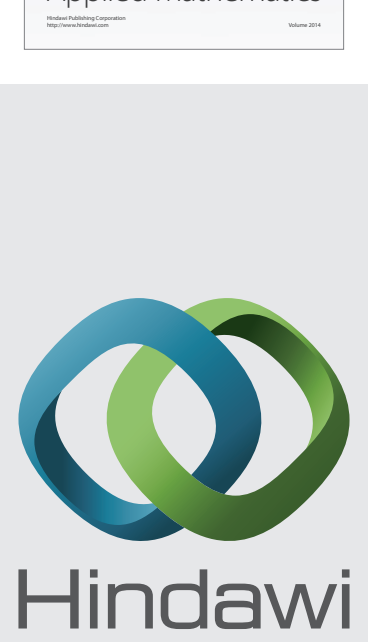

Submit your manuscripts at http://www.hindawi.com
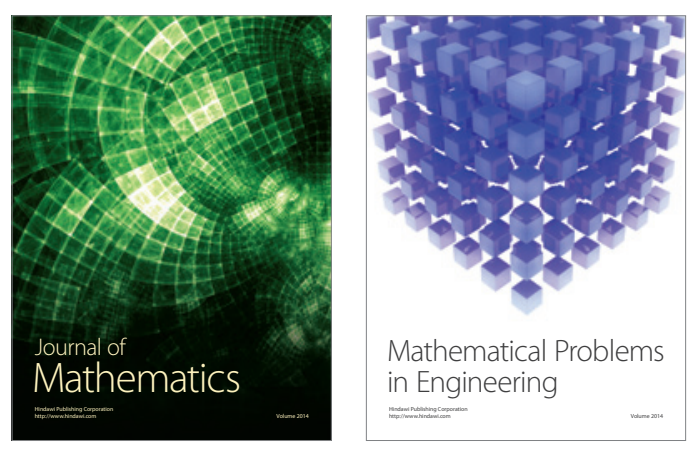

Mathematical Problems in Engineering
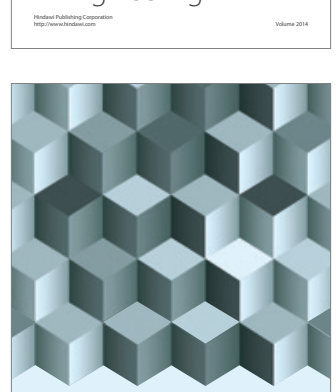

Journal of

Function Spaces
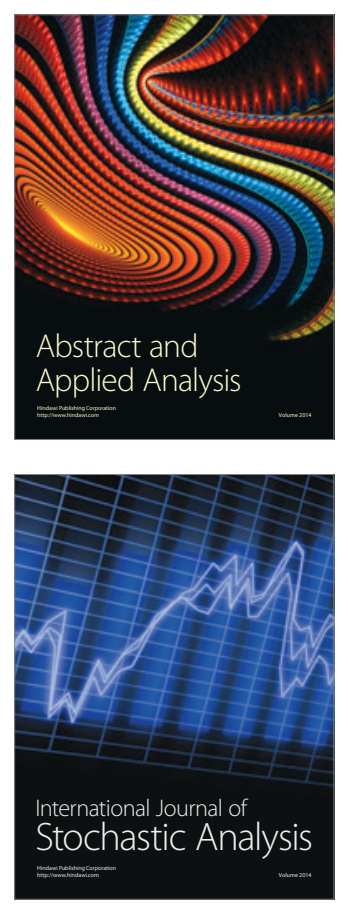

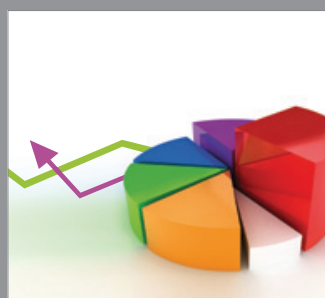

ournal of

Probability and Statistics

Promensencen
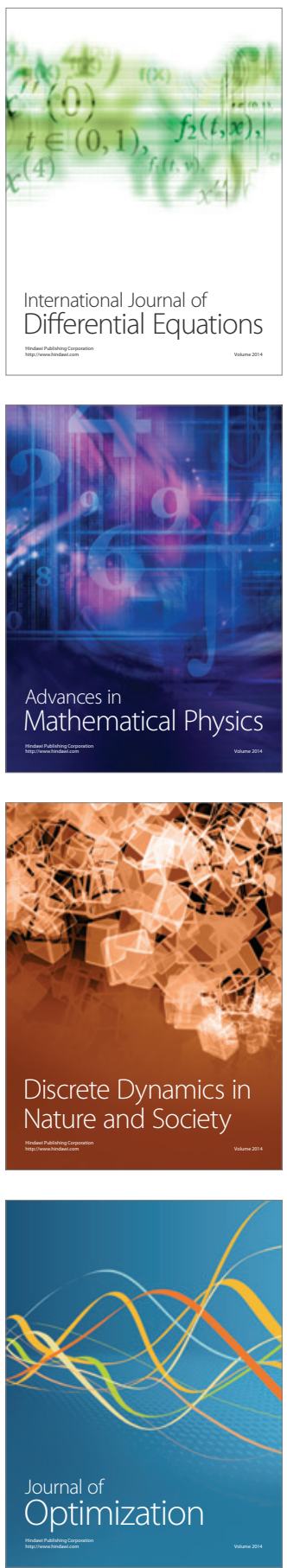